\title{
Genetic basis of progression and relapse in Clear Cell Sarcoma of the Kidney
}

\author{
Tomoki Yaguchi $^{1}$, Shunsuke Kimura ${ }^{1}$, Masafumi Seki ${ }^{1}$, Masahiro Sekiguchi ${ }^{1}$, Kenichi \\ Yoshida $^{2}$, Yuichi Shiraishi ${ }^{3}$, Keisuke Kataoka ${ }^{2}$, Yoichi Fujii ${ }^{2}$, Yasuo Kubota ${ }^{1}$, Kentaro \\ Watanabe $^{1}$, Mitsuteru Hiwatari ${ }^{1}$, Satoru Miyano ${ }^{4}$, Seishi Ogawa ${ }^{2}$, and Junko Takita ${ }^{2}$ \\ ${ }^{1}$ The University of Tokyo Graduate School of Medicine School of Medicine Department of \\ Pediatrics \\ ${ }^{2}$ Kyoto University Graduate School of Medicine Faculty of Medicine \\ ${ }^{3}$ National Cancer Center Research Institute \\ ${ }^{4}$ The University of Tokyo Institute of Medical Science Human Genome Center
}

November 27, 2020

\begin{abstract}
To elucidate the genetic mechanisms of recurrence and metastasis in clear cell sarcoma of the kidney (CCSK), a dismal pediatric renal cancer, we performed targeted-capture sequencing by pediatric solid tumors panel (381 genes) for multi-sampled tumors including autopsy samples of metastasis extracted from a single case that underwent four relapses. Internal tandem duplication of BCOR (BCOR-ITD) was the only truncal mutation, indicating a strong association with tumorigenesis. Acquisition of additional mutations along tumor relapses and detection of metastasis-specific mutations were reminiscent of the tumor progression and therapeutic resistance of this case, leading to clonal selection and a dismal fate.
\end{abstract}

\section{INTRODUCTION}

Clear cell sarcoma of the kidney (CCSK) makes up $4 \%$ of all primary renal malignancies in children ${ }^{1}$. Although its prognosis has significantly improved recently ${ }^{1-5}$, the relapse rate is still high and the prognosis of patients with relapses is extremely poor ${ }^{6}$. Internal tandem duplication of $B C O R(B C O R \text {-ITD })^{7,8}, Y W H A E$ NUTM2B/E fusion ${ }^{9,10}$, and BCOR -CCNB3 fusion ${ }^{11}$ have been reported in CCSK cases; however, the genetic mechanisms associated with tumor recurrence and metastasis are still poorly understood. In this study, we assessed the genetic mechanisms of CCSK recurrence and metastasis by analyzing multi-sampled tumors extracted from a single case: specimens at diagnosis, each relapse and autopsy.

\section{CASE REPORT}

A 2-year-old boy was diagnosed with CCSK, which relapsed 4 times until he yielded to disease at the age of 7 years (Fig. 1, Supplementary Fig. S1). At diagnosis, the primary tumor site was the right kidney (StageI). He received chemotherapy consisting of actinomycin D, vincristine and, doxorubicin (DD-4A) followed by radiotherapy $(10.8 \mathrm{~Gy} / 6 \mathrm{Fr})$ after a right partial nephrectomy. This combination therapy successfully induced complete remission. However, 4 months after the completion of treatment, first relapse occurred at his left lung. He received 6 courses of chemotherapy consisting of ifosfamide, carboplatin, and etoposide (ICE) with radiotherapy $(12 \mathrm{~Gy} / 8 \mathrm{Fr})$ after a left upper lobectomy, and then achieved second remission. At the age of 5 years, second relapse developed at the abdominal lymph nodes. A total excision was done after three courses of chemotherapy consisting of cyclophosphamide, vincristine, pirarubicin, and cisplatin. Postoperative chemoradiotherapy induced third remission. At the age of 6 years, he suffered from third 
relapse at the para-aortic lymph nodes. The patient underwent a subtotal excision of these lymph nodes followed by high dose chemotherapy (busulfan and melpharan) with autologous hematopoietic stem cell transplantation (aHSCT). The patient achieved fourth remission; however, four months later, fourth relapse occurred at the ascending colon, small intestine, duodenum, pancreas, and right hepatic lobe, and he yielded to the advanced disease.

\section{RESULTS}

After obtaining informed consent from his parents, DNA was extracted from the peripheral blood and biopsy samples of the tumors at initial diagnosis (D), each relapse (second relapse; R2, third relapse; R3, fourth relapse; R4) and autopsy (abdominal lymph node; LN, liver; Liver) except for the sample at first relapse. Direct sequencing identified BCOR -ITD (c.5526_5621dup) at all the analyzed samples (Supplementary Fig. S2). SNP array analysis did not reveal any copy number alterations (CNAs) at D whereas consistent CNAs on chromosomes 1q, 7q, and 15q were detected (Supplementary Fig. S3). Each sample possessed several sample specific CNAs. Moreover, we detected a focal loss at chromosome 9q32-33 in the samples of R2 through autopsy, where only the tumor suppressor gene BRINP1 was located (Fig. 2A). We further analyzed other genetic alterations using targeted-capture sequencing (TCS) for 381 genes relating to pediatric solid tumors $^{12}$. Candidate mutations were filtered according to our previous report ${ }^{12}$. Detected 13 mutations were classified into 7 clones based on the detected samples and variant allele frequency. Only the $B C O R$ -ITD was included in clone 1, whereas 3 clade mutations (FLT1, JAK2 , and $M Y H 7$ ) were additionally detected in relapsed tumor samples (clone 2, 3). The other mutations (PRKRIR, KMT2D , TSHR , CDK6 ,IL6ST , NF1 , KDM6A , OBSCN , and SYNE1) were clone specific (clone 4-7) (Supplementary Table S1). The number of detected mutations increased during each tumor recurrence. All R3 mutations (clone 1-3) were detected upon metastatic samples from autopsy, suggesting the accumulation of metastasis site specific mutations during tumor progression. Importantly, the newly acquired mutations at each relapse were not detected even as a minor clone in the previous samples. We analyzed the clonal evolution of the present case by using a software: clonevol $^{13,14}$. Multiregional sequencing analysis at different time points allowed us to delineate the clonal history of tumor cells, suggesting that each relapse developed based on the preexisting tumors (Fig. 2C).

\section{DISCUSSION}

In this study, we presented a case of CCSK which underwent 4 relapses. The first relapse occurred in the lung. This is consistent with the previous reports that the lung was the most common site of relapse in patients treated with DD- $4 \mathrm{~A}^{4,5}$, although the present case relapsed earlier (4 months vs 24 months $)^{1,3}$. The optimal treatment of relapsed CCSK has not been established as yet. ICE chemotherapy, a salvage regimen for recurrent $\mathrm{CCSK}^{6,15}$, resulted in the achievement of the second remission, however, the second relapse developed. Due to the refractory disease, we performed high dose chemotherapy with aHSCT rescue though its effectiveness is unclear ${ }^{6,15}$. Because treatment options for relapsed CCSK are limited and survival is poor, we retrospectively analyzed this case in terms of genomics for the possibility of new therapeutic strategies.

In this study, the same $B C O R$-ITD alteration was observed from diagnosis right up to autopsy with very low tumor mutation burden, suggesting a strong impact not only on CCSK tumorigenesis as previously reported $^{7,8}$ but also on its relapse. Meanwhile, we could not observe truncal mutations that can be targetable with available FDA-approved drugs. Multi-sample extractions at R4 and autopsy revealed site specific mutations of metastasis in addition to truncal mutations of R3 sample, which suggests the presence of subclones with site specific mutations in each metastases site of the previous samples. Among them, a subclone with selective advantage might relapse as the dominant clone. This is supported by the result that the newly acquired mutations were not detected even as a minor clone in the previous primary samples. Furthermore, our clonal analysis of the metastatic samples also suggested that relapsed tumors were derived from minor clones of the preceding tumors, which had survived chemoradiotherapy and thrived (Fig. 2C). Therefore, acquired genetic abnormalities during tumor progression might be associated with clinical therapeutic resistance ${ }^{16}$. BRINP1, lost in the samples of $\mathrm{R} 2$ through autopsy, is a tumor suppressor gene, which regulates the G1/S checkpoint and cell cycle ${ }^{17}$ and its dysregulation is associated with tumor recurrence 
in other carcinomas ${ }^{18}$. CNAs such as gain at $1 \mathrm{q}$ and loss at $7 \mathrm{q}$ and $15 \mathrm{q}$ are frequently observed in other neoplasms and related to tumor relapse and metastasis ${ }^{19-22}$. Moreover, acquired mutations in FLT1, JAK2 , $C D K 6$, NF1 , and KDM6A are generally associated with therapeutic resistance and metastasis ${ }^{23-30}$. Thus, we can estimate that the genetic alterations acquired during disease progression in the present case might contribute to clonal selection and therapeutic resistance. However, our analysis in the present study was limited in genomic abnormalities, and thus, further analyses for other various factors including epigenetic abnormalities and tumor microenvironment are warranted.

Altogether, although we failed to find targetable lesions, our study indicated the importance of $B C O R$-ITD in both CCSK tumorigenesis and relapse, and suggested that genetic alterations acquired during disease progression might contribute to clonal selection and therapeutic resistance in the present case. However, this report is based on a single case and further studies with more samples will help to detect common mechanisms of relapse and therapy resistance in CCSK, enabling appropriate therapy for relapsed cases considering genetic status.

\section{Conflict of Interest statement}

The authors declare no conflicts of interest.

\section{Acknowledgements}

The authors would like to thank Ms. M Matsumura, Ms. K Yin, and Ms. F Saito (The University of Tokyo) for their technical assistance.

This work was supported by Grants-in-Aid for Scientific Research from the Japan Society for the Promotion of Science (KAKENHI; grant nos. JP17H04224, JP18K19467, and JP20H00528 to J.T. and JP26221308 and JP19H05656 to S.O.); Project for Cancer Research and Therapeutic Evolution (P-CREATE; grant no. JP19cm0106509h9904 to J.T.), Project for Development of Innovative Research on Cancer Therapeutics (P-DIRECT; grant no. JP20cm0106501h0005 to S.O.), and Practical Research for Innovative Cancer Control (grant no. JP19ck0106468h0001 to J.T.) from Japan Agency for Medical Research and Development (AMED); Princess Takamatsu Cancer Research Fund (grant to J.T.).

\section{Authors' contribution}

TY, SK, MSeki, MSekig, and JT wrote the manuscript; TY, SK, MSeki and MSekig collected and analyzed data; TY, SK, MSekig, KY, YF, KK, YS, YK, KW, MH and SM performed experiments; SO and JT conceived the study; JT designed the study. All authors read and approved the final manuscript.

\section{Ethics approval and consent to participate}

The present study was approved by the Ethics Committee of the University of Tokyo.

\section{References}

1. P Argani, et al. Clear cell sarcoma of the kidney: a review of 351 cases from the National Wilms Tumor Study Group Pathology Center. Am J Surg Pathol 2000 Jan;24(1):4-18.

2. R. Furtwangler, et al. Clear Cell Sarcomas of the Kidney registered on International Society of Pediatric Oncology (SIOP) 93-01 and SIOP 2001 protocols: A report of the SIOP Renal Tumour Study Group. Eur J Cancer 2013;49:3497-3506.

3. S. L. Gooskens, et al. Clear cell sarcoma of the kidney: A review. Eur J Cancer 2012;48:2219-2226.

4. Nita L. Seibel, et al. Effect of Duration of Treatment on Treatment Outcome for Patients With ClearCell Sarcoma of the Kidney: A Report From the National Wilms' Tumor Study Group. J Clin Oncol 2004;22(3):468-73.

5. Nita L. Seibel, et al. Impact of cyclophosphamide and etoposide on outcome of clear cell sarcoma of the kidney treated on the National Wilms Tumor Study-5 (NWTS-5). Pediatr Blood Cancer 2019;66(1):e27450. 
6. S. L. Gooskens, et al. Treatment and outcome of patients with relapsed clear cell sarcoma of the kidney: a combined SIOP and AIEOP study. Br J Cancer 2014;111:227-233.

7. Ueno-Yokohata H, et al. Consistent in-frame internal tandem duplications of BCOR characterize clear cell sarcoma of the kidney. Nat Genet 2015;47(8):861-3.

8. Angshumoy Roy, et al. Recurrent internal tandem duplications of BCOR in clear cell sarcoma of the kidney. Nature Commun 2015;6:8891.

9. O'Meara E, et al. Characterization of the chromosomal translocation $\mathrm{t}(10 ; 17)(\mathrm{q} 22 ; \mathrm{p} 13)$ in clear cell sarcoma of kidney. J Pathol 2012;227(1):72-80.

10. André Fehr, et al. YWHAE-FAM22 gene fusion in clear cell sarcoma of the kidney. J Pathol 2012;227:5-7.

11. Meng K Wong, et al. Clear cell sarcomas of the kidney are characterised by BCOR gene abnormalities, including exon 15 internal tandem duplications and BCOR-CCNB3 gene fusion. Histopathology 2018;72:320329 .

12. Masahiro Sekiguchi, et al. Integrated multiomics analysis of hepatoblastoma unravels its heterogeneity and provides novel druggable targets. NPJ Precis Oncol. 2020;4:20.

13. Dang HX, et al. ClonEvol: clonal ordering and visualization in cancer sequencing. Ann Oncol 2017;28(12):3076-3082.

14. Shunsuke Kimura, et al. NOTCH 1 pathway activating mutations and clonal evolution in pediatric T-cell acute lymphoblastic leukemia. Cancer Sci 2019;110:784-794.

15. Vlad C, et al. Treatment of recurrent clear cell sarcoma of the kidney with brain metastasis. Pediatr Blood Cancer 2008;50(2):246-9.

16. Mossner M, et al. Mutational hierarchies in myelodysplastic syndromes dynamically adapt and evolve upon therapy response and failure. Blood 2016;128(9):1246-59.

17. Hiroyuki Nishiyama, et al. Negative regulation of G1/S transition by the candidate bladder tumour suppressor gene DBCCR1. Oncogene 2001;20:2956-2964.

18. A Lopez-Beltran, et al. Loss of heterozygosity at 9q32-33 (DBC1 locus) in primary non-invasive papillary urothelial neoplasm of low malignant potential and low-grade urothelial carcinoma of the bladder and their associated normal urothelium. J Pathol 2008;215:263-272.

19. Eric J Gratias, et al. Gain of 1q is Associated with Inferior Event-Free and Overall Survival in Favorable Histology Wilms Tumor: A Report from the Children's Oncology Group. Cancer 2013;119:3887-3894.

20. H Kuniyasu, et al. Frequent loss of heterozygosity of the long arm of chromosome 7 is closely associated with progression of human gastric carcinomas. Int J Cancer 1994;59:59-600.

21. Poetsch M, et al. Loss of heterozygosity at $15 q 21.3$ correlates with occurrence of metastases in head and neck cancer. Mod Pathol 2006;19(11):1462-9.

22. Rhiem K, et al. Chromosomal region $15 q 21.1$ is a frequent target of allelic imbalance in advanced breast carcinomas. Int J Cancer 2003;106(1):74-7.

23. Evert Jan Van Limbergen, et al. FLT1 kinase is a mediator of radioresistance and survival in head and neck squamous cell carcinoma. Acta Oncol 2014;53:637-645.

24. Bin-Zhi Qian, et al. FLT1 signaling in metastasis-associated macrophages activates an inflammatory signature that promotes breast cancer metastasis. J Exp Med 2015;212:1433-1448.

25. Vainchenker W, et al. JAK/STAT signaling in hematological malignancies. Oncogene 2013;32:2601-13. 
26. Chloe James, et al. A unique clonal JAK2 mutation leading to constitutive signaling causes polycythaemia vera. Nature 2005;434: 1144-8.

27. Alexandra Wolf, et al. JAK2-V617F-induced MAPK activity is regulated by PI3K and acts synergistically with PI3K on the proliferation of JAK2-V617F-positive cells. JAKSTAT 2013;2;e24574.

28. Li F, et al. YAP1-Mediated CDK6 Activation Confers Radiation Resistance in Esophageal Cancer Rationale for the Combination of YAP1 and CDK4/6 Inhibitors in Esophageal Cancer. Clin Cancer Res 2019;25(7):2264-2277.

29. Kurimchak A M, et al. Intrinsic Resistance to MEK Inhibition through BET Protein-Mediated Kinome Reprogramming in NF1-Deficient Ovarian Cancer. Mol Cancer Res 2019;17(8):1721-1734.

30. Sophie M. Stief, et al. Loss of KDM6A confers drug resistance in acute myeloid leukemia. Leukemia 2020;34:50-62.

\section{Figure Legends}

Figure 1. Clinical presentation in this case. Each mutation detected by targeted-capture sequencing was classified based on the detected samples and variant allele frequency, while there were no data available for clone at first relapse. Estimated cell population at each sample is shown. Tumor at third and fourth relapse showed therapeutic resistance.

Figure 2. A; The results of copy number analysis of SNP array for chromosome 9 . Red dots at the top represent the signal from each probe (raw data), and blue line indicates the moving average of red dots. Red and green lines at the bottom represent allele-specific copy number. Allelic loss of chromosome 9q including BRINP1 gene was observed in R2, R3, R4, LN, and Liver samples.

B; Each mutation detected by TCS was classified into 7 clones according to its variant allele frequency (VAF). VAFs of mutations and estimated cell population of each sample are shown.

C; The schematic representation of clonal evolution constructed from somatic mutations detected by TCS. $B C O R$-ITD was the only truncal mutation detected in both primary and relapse samples. Additional mutations accumulated during disease progression were shown.

CN, copy number; D, initial diagnosis; R2, second relapse; R3, third relapse; R4, fourth relapse; LN, lymph node; VAF, variant allele frequency.

Supplementary Figure 1. Computed tomography imaging during the clinical course of the present case, A; at initial diagnosis, B; at first relapse, C; at second relapse, D; at third relapse, E, F, G, H, and I; at fourth relapse. White arrows represent tumor site.

Supplementary Figure 2. A; BCOR -ITD (c.5296_5391dup; NM_0177455) was detected from all tumor samples except R1. The reference sequence (top) and the sequence data obtained from primary sample (bottom) are shown. The parental (red) and duplicated (blue) segments are indicated.

B; Genomic PCR analysis of BCOR exon 15. The PCR products from samples in D, R1, R2, R3, R4, LN, and Liver are presented. Targeted PCR and gel electrophoresis of BCOR exon 15 in samples from D, R2, R3, LN and Liver showed large product.

C; Schema representing structural variant of BCOR -ITD. Parental segment in PUFD domain was duplicated (ITD).

M, marker: C, control (peripheral blood); D, initial diagnosis; R2, second relapse; R3, third relapse; R4, fourth relapse; LN, lymph node; ANK, ankyrin repeats; PUFD, PCGF ubiquitin-like fold discriminator.

Supplementary Figure 3. Results of SNP array analysis in each sample. Colorful dots at the top represent signal of each probe (raw data). Red and green lines at the bottom show allele specific copy number, respectively. 
D, initial diagnosis; R2, second relapse; R3, third relapse; R4, fourth relapse; LN, lymph node.

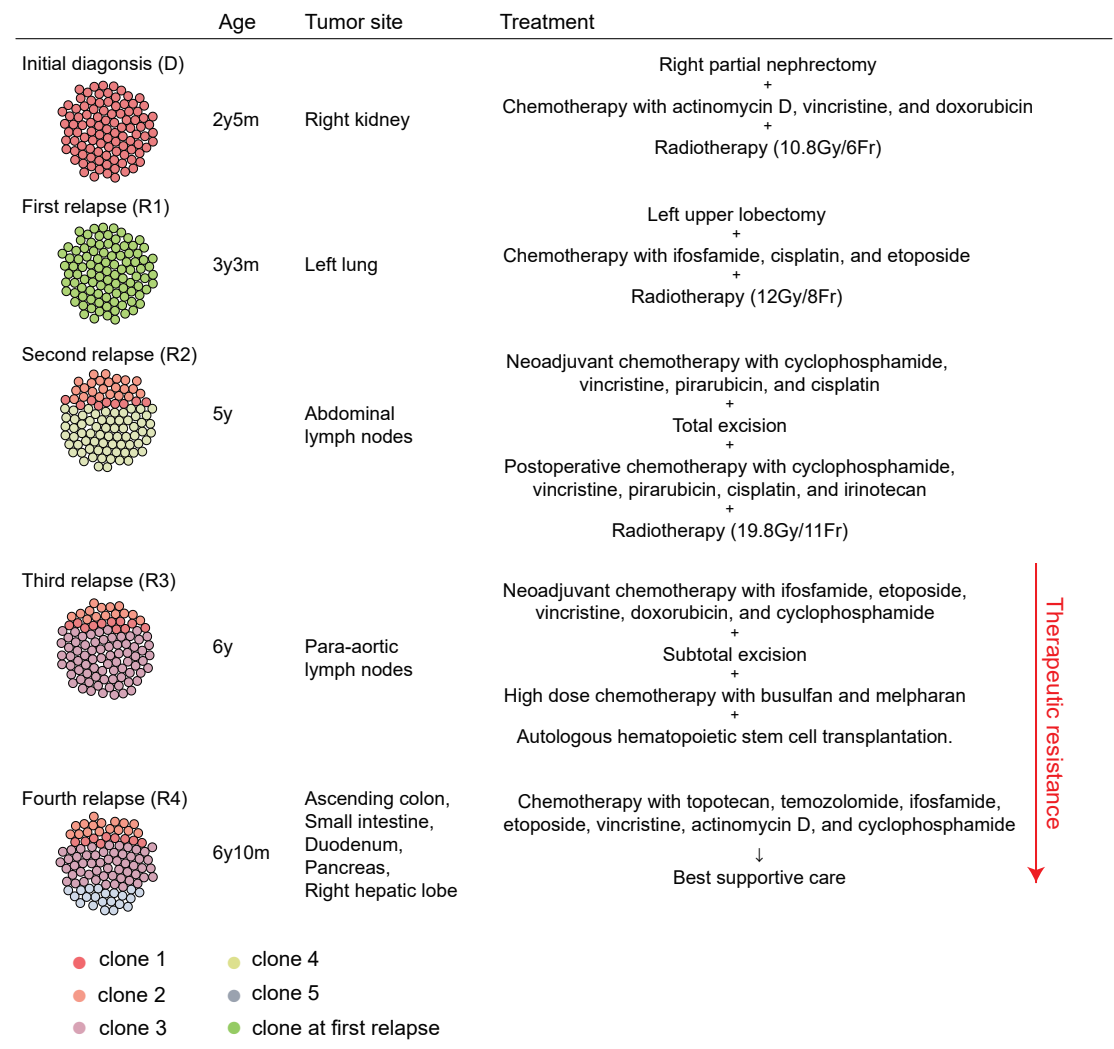


A

Chr9q(R2)
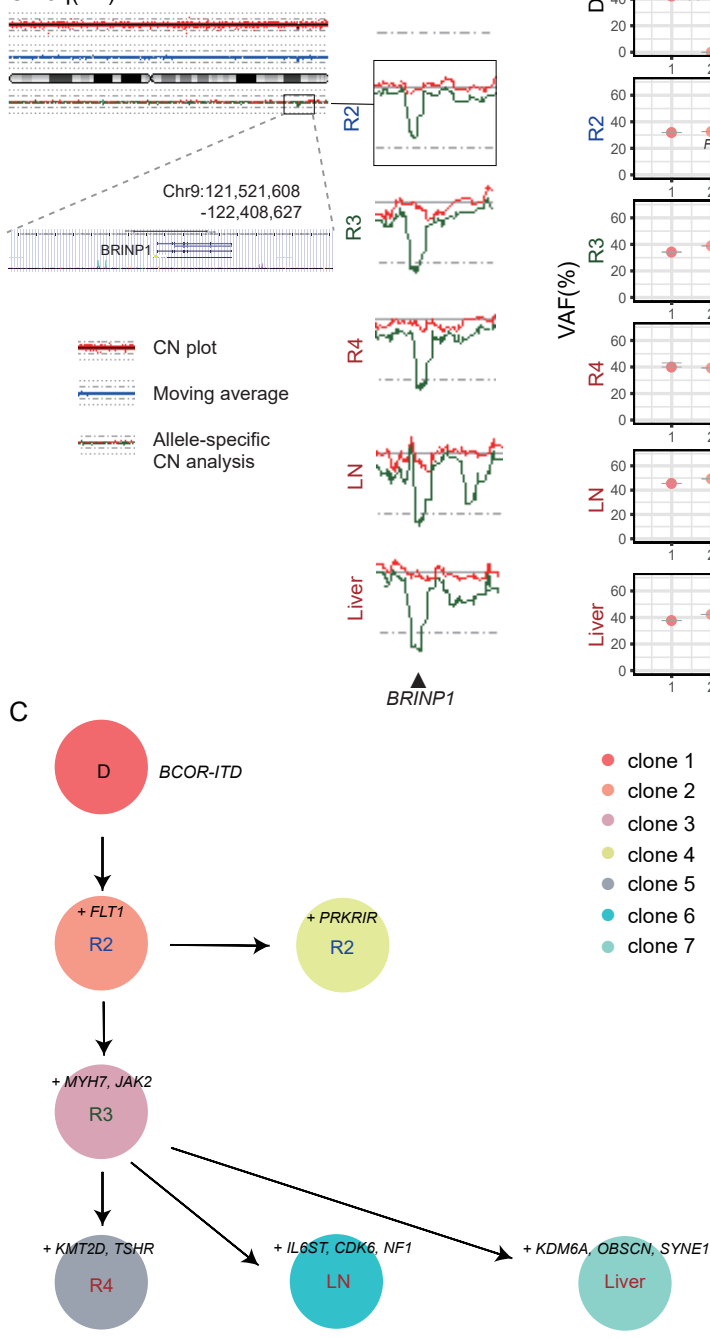

B

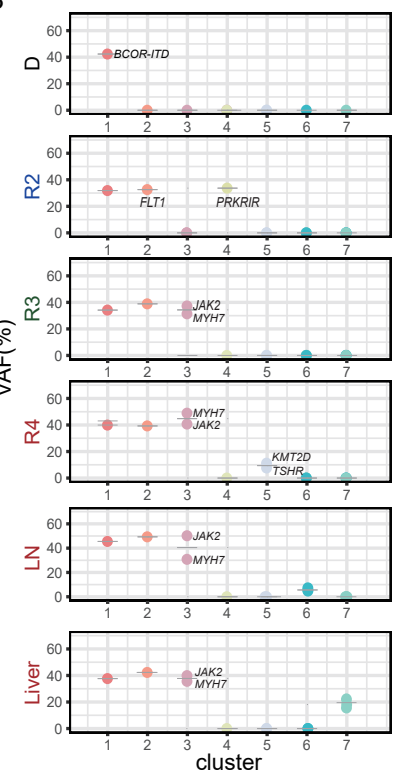

- clone 1

clone 2

clone 5

Liver 